\title{
Design, Synthesis, Spectroscopic Characterization of New Pyrazole Carbothioamides as Antifungal and Antibacterial Candidates
}

\author{
Deepashree Nagaraj ${ }^{1}\left(\mathbb{D}\right.$, Renuka Nagamallu ${ }^{2}$ (D), Ajay Kumar Kariyappa $1, * \mathbb{C}$ \\ Department of Chemistry, Yuvaraja College, University of Mysore, Mysuru-570005, India \\ Department of Chemistry, GSSS College of Engineering and Technology for Women, Mysuru, India \\ * Correspondence: ajaykumar@ycm.uni-mysore.ac.in (A.K.K.);
}

Scopus Author ID 6506781846

Received: 28.04.2021; Revised: 25.06.2021; Accepted: 3.07.2021; Published: 7.09.2021

\begin{abstract}
In a sustained search for novel antimicrobial agents as weaponry in the war against infectious diseases, resulting in improved survivability for both humans and their domestic animals, the present study demonstrates an efficient synthesis of $N, N$-dimethylaminophenyl substituted pyrazole carbothioamide derivatives. The synthesis involves $(3+2)$ cycloaddition of chalcones with hydrazinecarbothioamide hydrochloride in the presence of the amberlyst-15 catalyzed at room temperature. The structures of new compounds were characterized by spectroscopic analysis. Furthermore, the synthesized new compounds $5(\mathrm{a}-\mathrm{g})$ were assessed in vitro for their antimicrobial susceptibilities. The results indicate that compounds $5 \mathrm{a}$ found potent against tested bacteria species; $5 \mathrm{~b}$ and $5 \mathrm{c}$ show excellent inhibition against the tested fungi and bacteria species. Therefore, these could act as antifungal and antibacterial leads for further investigations.
\end{abstract}

Keywords: annulation; antimicrobial; chalcones; cycloaddition; minimum inhibition.

(C) 2021 by the authors. This article is an open-access article distributed under the terms and conditions of the Creative Commons Attribution (CC BY) license (https://creativecommons.org/licenses/by/4.0/).

\section{Introduction}

The discovery of new antimicrobials with greater efficacy and lesser toxicity is a huge revolutionary task for researchers all over. This is because the subsequent realization immediately ebbed the health triumph that bacterial populations could quickly modify themselves to resist antimicrobials, propagate these resistance traits, and even share resistance genes with other contemporary bacteria within their environment [1]. The compounds with pyrazole core are important pharmacophores in the development of antimicrobials, they alone or combined with other pharmacophores display potent antimicrobial activity [2]. The discovery of antipyrine by Knorr led to wide openings for scientists to work in the field of pyrazoles. A silver-mediated cycloaddition of N-isocyanoiminotriphenylphosphorane synthesized the pyrazole derivatives to terminal alkynes under mild reaction conditions [3], one-pot synthesis from ketones, aldehydes, and hydrazines [4], 1,3-dipolar cycloaddition of aldehyde hydrazones to alkenes in the presence of chloramine-T as dehydrogenating agent [57], and a phosphine-free [3+2] cycloaddition of dialkyl azodicarboxylates to propargyl amines [8]. The regioselective synthesis of pyrazoles was achieved by ruthenium $\left[\mathrm{RuH}_{2}\left(\mathrm{PPh}_{3}\right)_{3} \mathrm{CO}\right] /$ Xanthphos catalyzed reaction of 1,3-diols and alkyl hydrazines [9]. An acid-mediated $(3+2)$ annulation reaction of chalcones with hydrazines [10-13], semicarbazide [14-16], and thiosemicarbazide [17-20] produce pyrazole derivatives with high regioselectivity. 
Chalcones involve the biosynthesis of flavonoids and isoflavonoids [21] and are useful intermediates in the synthesis of pyrazole derivatives [22, 23]. The pyrazole analogs are found as versatile pharmacophores for their pharmaceutical utilities. The molecules with pyrazole skeleton have reported to; inhibit the growth of DLA cells in vitro by committing them towards apoptosis [24], inhibit $\alpha$-glucosidase [25], 15-LOX [26], selective BuChE [27], metallo- $\beta$ lactamase [28], phospholipase A2 [29, 30], and CDK2 [31]. These classes of compounds also known to exhibit anticancer [32, 33], angiogenic [34], anti-inflammatory [35], and antioxidant $[36,37]$ properties. Extensive studies on the antimicrobial properties of pyrazole derivatives have been reported $[37,38]$. Overall, the modifications of the pyrazole core with varied substitutions could enhance the antimicrobial inhibition potentials. In this pretext, aiming to build up more antimicrobial active templates, a series of pyrazole carbothioamides, $5(\mathrm{a}-\mathrm{g})$, were synthesized through a simple approach. After the structural confirmations, the compounds were examined for their in vitro antimicrobial susceptibilities.

\section{Materials and Methods}

\subsection{Synthesis of chalcones, 3(a-g).}

To a solution mixture of 4-(dimethylamino)benzaldehyde $1(10 \mathrm{mmol})$ and substituted acetophenones 2(a-g) $(10 \mathrm{mmol})$ in methyl alcohol, potassium hydroxide solution $(40 \%, 2 \mathrm{~mL})$ was added. Then the solution mixture was stirred at room temperature for 3-4 h. After completion, the mixture was cooled to room temperature, poured into ice-cold water, and kept overnight in the refrigerator. The solids separated were filtered, washed successively with cold hydrochloric acid (5\%) and cold water. The crude solids were recrystallized from methyl alcohol to obtain compounds 3(a-g).

\subsection{Synthesis of pyrazole carbothioamide derivatives, 5(a-g).}

A mixture of chalcones 3(a-g) (5.0 mmol), hydrazinecarbothioamide hydrochloride, 4 (5 mmol), and amberlyst-15 (10\%, w/w) in acetonitrile $(25 \mathrm{~mL})$ was stirred at room temperature for 1-2 hr. The reactions were checked on thin-layer chromatography (TLC) plates pre-coated with silica gel using solvent system hexane: ethyl acetate (1:4) as eluent. After completion, the solid separated was filtered, washed by diethyl ether $(2 \times 20 \mathrm{~mL})$, dried, and treated with EtOAc $(20 \mathrm{~mL})$. After stirring for $10 \mathrm{~min}$, the mixture was filtered to remove the insoluble catalyst. The filtrate was collected and concentrated under a vacuum. The solid isolated was triturated in diethyl ether, filtered, and dried to obtain the desired products $5(\mathrm{a}-\mathrm{g})$.

Alternatively, a solution mixture of chalcones $3(\mathrm{a}-\mathrm{g})(5 \mathrm{mmol})$ and hydrazinecarbothioamide hydrochloride, $4(5.0 \mathrm{mmol})$ in acetic acid $(30 \%)$ was refluxed on a water bath for 2-3 h. After the completion, the mixture was filtered, and the filtrate was poured into crushed ice. The separated solids were filtered and washed successively with $5 \% \mathrm{NaHCO}_{3}$ and water. The crude solids were recrystallized from methyl alcohol to get $5(\mathrm{a}-\mathrm{g})$.

\subsection{Antimicrobial activity.}

The antimicrobial activities of the compounds 5(a-g) were determined as minimum inhibitory concentrations (MIC) by the serial dilution method [40, 41]. The antibacterial tests were conducted against bacterial pathogens like Escherichia coli (MTCC 1687), Bacillus subtilis (MTCC 441), and Staphylococcus aureus (MTCC 737), and antifungal activity against 
Aspergillus niger, Aspergillus flavus, and Candida albicans (MTCC 227) stains. The antibiotics ciprofloxacin and nystatin were used as a positive control against bacterial and fungal species, respectively, and dimethyl sulfoxide was used as solvent control. The experiments were conducted in triplicate; the results were taken as a mean \pm variance (SD).

\section{Results and Discussion}

\subsection{Analytical data.}

3.1.1. 3-(4-(Dimethylamino)phenyl)-1-phenylprop-2-en-1-one, 3.

Obtained from 4-(dimethylamino)benzaldehyde, $1(1.49 \mathrm{~g}, 10 \mathrm{mmol})$ and acetophenone, 2a $(1.20 \mathrm{~g}, 10 \mathrm{mmol})$ in $72 \%$ yield, m.p. $102-104{ }^{\circ} \mathrm{C} ;{ }^{1} \mathrm{H} \mathrm{NMR}\left(\mathrm{CDCl}_{3}, \delta \mathrm{ppm}\right): 3.010(\mathrm{~s}, 6 \mathrm{H}$, $\left.\mathrm{CH}_{3}\right), 6.721(\mathrm{~d}, 1 \mathrm{H}, J=16.6 \mathrm{MHz},=\mathrm{CH}), 6.998-7.250(\mathrm{~m}, 6 \mathrm{H}, \mathrm{Ar}-\mathrm{H}), 7.465-7.534(\mathrm{~m}, 3 \mathrm{H}, \mathrm{Ar}-$ $\mathrm{H}), 8.245(\mathrm{~d}, 1 \mathrm{H}, J=15.0 \mathrm{MHz}, \mathrm{CH}=) ;{ }^{13} \mathrm{C} \mathrm{NMR}\left(\mathrm{CDCl}_{3}, \delta \mathrm{ppm}\right): 40.2\left(2 \mathrm{C}, \mathrm{N}\left(\mathrm{CH}_{3}\right)_{2}\right), 111.0$ (2C), 121.4 (1C), 124.6 (1C), 128.1 (1C), 128.3 (1C), 128.5 (1C), 129.3 (1C), 129.7 (2C), 134.3 (1C), 136.0 (1C), 143.4 (1C), 153.0 (1C), 188.3 (1C, C=O); MS m/z: 251.13 (M+, 100); Anal. Calcd. (found) for $\mathrm{C}_{17} \mathrm{H}_{17} \mathrm{NO}(\%)$ : C, 81.24 (81.17); H, 6.82 (6.78); N, 5.57 (5.49).

\subsubsection{3-(4-(Dimethylamino)phenyl)-1-(4-fluorophenyl)prop-2-en-1-one, 3b.}

Obtained from 4-(dimethylamino)benzaldehyde, 1 (1.49g, $10 \mathrm{mmol})$ and 1-(4fluorophenyl)ethan-1-one, $2 \mathrm{~b}(1.38 \mathrm{~g}, 10 \mathrm{mmol})$ in $68 \%$ yield, m.p. $94-95{ }^{\circ} \mathrm{C} ;{ }^{1} \mathrm{H}$ NMR (DMSO-d 6 , $\delta$ ppm): $3.005\left(\mathrm{~s}, 6 \mathrm{H}, \mathrm{CH}_{3}\right), 6.898(\mathrm{~d}, 1 \mathrm{H}, \mathrm{J}=17.2 \mathrm{MHz},=\mathrm{CH}), 7.103-7.303(\mathrm{~m}$, $4 \mathrm{H}, \mathrm{Ar}-\mathrm{H}), 7.378-7.596(\mathrm{~m}, 4 \mathrm{H}, \mathrm{Ar}-\mathrm{H}), 8.355$ (d, 1H, J=15.2MHz, CH=); ${ }^{13} \mathrm{C}$ NMR (DMSO$\left.\mathrm{d}_{6}, \delta \mathrm{ppm}\right): 39.5\left(2 \mathrm{C}, \mathrm{N}\left(\mathrm{CH}_{3}\right)_{2}\right), 111.6(2 \mathrm{C}), 114.8(2 \mathrm{C}), 124.4(1 \mathrm{C}), 128.9(2 \mathrm{C}), 129.0(1 \mathrm{C})$, 129.6 (1C), 136.0 (1C), 143.1 (1C), 143.4 (1C), 153.2 (1C), 167.1 (1C), 187.2 (1C, C=O); MS (m/z): $269.1(\mathrm{M}+, 100)$; Anal. Calcd. (found) for $\mathrm{C}_{17} \mathrm{H}_{16} \mathrm{FNO}(\%)$ : 75.82 (75.74); H, 5.99 (5.94); N, 5.20 (5.15).

\subsubsection{1-(4-Chlorophenyl)-3-(4-(dimethylamino)phenyl)prop-2-en-1-one, 3c.}

Obtained from 4-(dimethylamino)benzaldehyde, $1(1.49 \mathrm{~g}, 10 \mathrm{mmol})$ and 1-(4chlororophenyl)ethan-1-one, 2c (1.54g, $10 \mathrm{mmol})$ in $77 \%$ yield, m.p. $135-136{ }^{\circ} \mathrm{C} ;{ }^{1} \mathrm{H}$ NMR $\left(\mathrm{CDCl}_{3}, \delta \mathrm{ppm}\right): 3.010\left(\mathrm{~s}, 6 \mathrm{H}, \mathrm{CH}_{3}\right), 6.956(\mathrm{~d}, 1 \mathrm{H}, \mathrm{J}=16.1 \mathrm{MHz},=\mathrm{CH}), 7.012-7.233(\mathrm{~m}, 4 \mathrm{H}$, Ar-H), 7.560-7.788 (m, 4H, Ar-H), $8.205(\mathrm{~d}, 1 \mathrm{H}, \mathrm{J}=17.8 \mathrm{MHz}, \mathrm{CH}=) ;{ }^{13} \mathrm{C} \mathrm{NMR}\left(\mathrm{CDCl}_{3}, \delta\right.$ ppm): 40.2 (2C, $\left.\mathrm{N}\left(\mathrm{CH}_{3}\right)_{2}\right), 112.8$ (2C), 121.2 (1C), 124.8 (1C), 127.2 (2C), 129.1 (2C), 130.0 (2C), 135.8 (1C), 140.2 (1C), 144.5 (1C), 149.6 (1C), 188.1 (1C, C=O); MS (m/z): $287.2(\mathrm{M}+2$, 32), $285.1(\mathrm{M}+, 100)$; Anal. Calcd. (found) for $\mathrm{C}_{17} \mathrm{H}_{16} \mathrm{ClNO}(\%)$ : C, 71.45 (75.36); H, 5.64 (5.61); N, 4.90 (4.87).

\subsubsection{3-(4-(Dimethylamino)phenyl)-1-(p-tolyl)prop-2-en-1-one, 4c.}

The analytical data of the compound were in good agreement with the previous report [39].

3.1.5. 3-(4-(Dimethylamino)phenyl)-1-(3-methoxyphenyl)prop-2-en-1-one, 3e.

Obtained from 4-(dimethylamino)benzaldehyde, 1 (1.49g, $10 \mathrm{mmol})$ and 1-(3methoxyphenyl)ethan-1-one, 2e (1.50g, $10 \mathrm{mmol})$ in $61 \%$ yield, m.p. $119-121{ }^{\circ} \mathrm{C} ;{ }^{1} \mathrm{H}$ NMR 
$\left(\mathrm{CDCl}_{3}, \delta \mathrm{ppm}\right): 3.022\left(\mathrm{~s}, 6 \mathrm{H}, \mathrm{CH}_{3}\right), 3.810\left(\mathrm{~s}, 3 \mathrm{H}, \mathrm{OCH}_{3}\right), 6.992(\mathrm{~d}, 1 \mathrm{H}, \mathrm{J}=15.8 \mathrm{MHz},=\mathrm{CH})$, 7.009 (d, 2H, J= 11.3 MHz, Ar-H), 7.426-7.690 (m, 6H, Ar-H), 8.211 (d, 1H, J=13.5MHz, $\mathrm{CH}=) ;{ }^{13} \mathrm{C} \mathrm{NMR}\left(\mathrm{CDCl}_{3}, \delta \mathrm{ppm}\right): 41.2\left(2 \mathrm{C}, \mathrm{N}\left(\mathrm{CH}_{3}\right)_{2}\right), 55.4\left(1 \mathrm{C}, \mathrm{OCH}_{3}\right) 111.9(2 \mathrm{C}), 114.5$ (1C), 115.2 (1C), 120.1 (1C), 121.9 (1C), 125.2 (1C), 128.9 (2C), 130.0 (1C), 133.2 (1C), 144.6 (1C), 160.0 (1C), 148.8 (1C), 190.2 (1C, C=O); MS (m/z): 281.1 (M+, 100); Anal. Calcd. (found) for $\mathrm{C}_{18} \mathrm{H}_{19} \mathrm{NO}_{2}$ (\%): C, 76.84 (76.74); H, 6.81 (6.77); N, 4.98 (4.94).

3.1.6. 3-(4-(Dimethylamino)phenyl)-1-(4-methoxyphenyl)prop-2-en-1-one, 3f.

Obtained from 4-(dimethylamino)benzaldehyde, $1(1.49 \mathrm{~g}, 10 \mathrm{mmol})$ and 1-(4methoxyphenyl)ethan-1-one, $2 \mathrm{f}(1.50 \mathrm{~g}, 10 \mathrm{mmol})$ as semisolid in $66 \%$ yield; ${ }^{1} \mathrm{H} \mathrm{NMR}\left(\mathrm{CDCl}_{3}\right.$, $\delta \mathrm{ppm}): 3.010\left(\mathrm{~s}, 6 \mathrm{H}, \mathrm{CH}_{3}\right), 3.840\left(\mathrm{~s}, 3 \mathrm{H}, \mathrm{OCH}_{3}\right), 6.922(\mathrm{~d}, 1 \mathrm{H}, \mathrm{J}=14.6 \mathrm{MHz},=\mathrm{CH}), 7.022-$ $7.540(\mathrm{~m}, 6 \mathrm{H}, \mathrm{Ar}-\mathrm{H}), 7.745-7.782(\mathrm{~m}, 2 \mathrm{H}, \mathrm{Ar}-\mathrm{H}), 8.223$ (d, 1H, J=14.2MHz, CH=); ${ }^{13} \mathrm{C} \mathrm{NMR}$ $\left(\mathrm{CDCl}_{3}, \delta \mathrm{ppm}\right): 41.6\left(2 \mathrm{C}, \mathrm{N}\left(\mathrm{CH}_{3}\right)_{2}\right), 55.2\left(1 \mathrm{C}, \mathrm{OCH}_{3}\right) 111.8(2 \mathrm{C}), 114.0(2 \mathrm{C}), 121.4(1 \mathrm{C})$, 124.3 (1C), 128.7 (2C), 129.3 (2C), 131.4 (2C), 144.0 (1C), 150.0 (1C), 165.1 (1C), 189.5 (1C, $\mathrm{C}=\mathrm{O}$ ); Anal. Calcd. (found) for $\mathrm{C}_{18} \mathrm{H}_{19} \mathrm{NO}_{2}$ (\%): C, 76.84 (76.76); H, 6.81 (6.78); N, 4.98 (4.95).

\subsubsection{1-(3,4-Dimethoxyphenyl)-3-(4-(dimethylamino)phenyl)prop-2-en-1-one, 3g.}

Obtained from 4-(dimethylamino)benzaldehyde, $1(1.49 \mathrm{~g}, 10 \mathrm{mmol})$ and 1-(3,4dimethoxyphenyl)ethan-1-one, $2 \mathrm{~g}(1.80 \mathrm{~g}, 10 \mathrm{mmol})$ in $68 \%$ yield, m.p. $102-104{ }^{\circ} \mathrm{C} ;{ }^{1} \mathrm{H} \mathrm{NMR}$ $\left(\mathrm{CDCl}_{3}, \delta \mathrm{ppm}\right): 3.036\left(\mathrm{~s}, 6 \mathrm{H}, \mathrm{CH}_{3}\right), 3.845\left(\mathrm{~s}, 6 \mathrm{H}, \mathrm{OCH}_{3}\right), 6.975(\mathrm{~d}, 1 \mathrm{H}, \mathrm{J}=18.2 \mathrm{MHz},=\mathrm{CH})$, 6.990-7.212 (m, 4H, Ar-H), 7.734-7.847 (m, 3H, Ar-H), 8.312 (d, 1H, J=16.1MHz, CH=); ${ }^{13} \mathrm{C}$ NMR $\left(\mathrm{CDCl}_{3}, \delta\right.$ ppm): $41.1\left(2 \mathrm{C}, \mathrm{N}\left(\mathrm{CH}_{3}\right)_{2}\right), 55.4\left(2 \mathrm{C}, \mathrm{OCH}_{3}\right) 108.4(1 \mathrm{C}), 112.5(2 \mathrm{C}), 113.3$ (1C), 120.8 (1C), 122.9 (1C), 123.4 (1C), 124.7 (1C), 129.5 (2C), 144.8 (1C), 149.6 (1C), 150.7 (1C), 154.1 (1C), 190.1 (1C, C=O); MS (m/z): 311.1 (M+, 100); Anal. Calcd. (found) for $\mathrm{C}_{19} \mathrm{H}_{21} \mathrm{NO}_{3}(\%)$ : C, 73.29 (73.22); H, 6.80 (6.76); N, 4.50 (4.46).

3.1.8. 5-(4-(Dimethylamino)phenyl)-3-phenyl-4,5-dihydro-1H-pyrazole-1-carbothioamide, 5a.

Obtained from 3-(4-(dimethylamino)phenyl)-1-phenylprop-2-en-1-one, 3a (1.75g, 5 mmol) and hydrazinecarbothioamide hydrochloride, 4 (0.64g, $5 \mathrm{mmol}) ;{ }^{1} \mathrm{H} \mathrm{NMR}\left(\mathrm{CDCl}_{3}, \delta\right.$ ppm): 3.005 (s, 6H, CH $\mathrm{CH}_{3}$ ) 3.655 (dd, 1H, J=9.6, 17.2Hz, 4- $\mathrm{H}_{\mathrm{a}}$ ), 3.965 (dd, 1H, J=19.4, 6.6Hz, 4-H $\mathrm{H}_{\mathrm{b}}, 4.304$ (dd, 1H, J=11.8, 7.0Hz, C5-H), 6.874-7.008 (m, 4H, Ar-H), 7.435-7.691 (m, 5H, Ar-H), 8.345 (s, 2H, NH$) ;{ }^{13} \mathrm{C} \mathrm{NMR}\left(\mathrm{CDCl}_{3}, \delta \mathrm{ppm}\right): 41.8\left(2 \mathrm{C}, \mathrm{N}\left(\mathrm{CH}_{3}\right)_{2}\right), 43.8(1 \mathrm{C}, \mathrm{C}-4)$, 69.9 (1C, C-5), 113.1 (2C), 128.1 (2C), 128.6 (2C), 129.4 (2C), 131.6 (1C), 132.6 (1C), 137.0 (1C), 149.0 (1C), 152.7 (1C, C-3), 175.1 (1C, C=S); MS (m/z): 324.1 (M+, 100); Anal. Calcd. (found) for $\mathrm{C}_{18} \mathrm{H}_{20} \mathrm{~N}_{4} \mathrm{~S}$ (\%): C, 66.64 (66.53); H, 6.21 (6.17); N, 17.27 (17.22).

3.1.9. 5-(4-(Dimethylamino)phenyl)-3-(4-fluorophenyl)-4,5-dihydro-1H-pyrazole-1carbothioamide, $5 \mathrm{~b}$.

Obtained from 3-(4-(dimethylamino)phenyl)-1-(4-fluorophenyl)prop-2-en-1-one, 3b $(1.35 \mathrm{~g}, 5 \mathrm{mmol})$ and hydrazinecarbothioamide hydrochloride, $4(0.64 \mathrm{~g}, 5 \mathrm{mmol}) ;{ }^{1} \mathrm{H}$ NMR $\left(\mathrm{CDCl}_{3}, \delta \mathrm{ppm}\right): 2.990\left(\mathrm{~s}, 6 \mathrm{H}, \mathrm{CH}_{3}\right), 3.614\left(\mathrm{dd}, 1 \mathrm{H}, \mathrm{J}=10.5,17.4 \mathrm{MHz}, 4-\mathrm{H}_{\mathrm{a}}\right), 3.910(\mathrm{dd}, 1 \mathrm{H}$, $\left.\mathrm{J}=19.2,7.6 \mathrm{MHz}, 4-\mathrm{H}_{\mathrm{b}}\right), 4.215(\mathrm{dd}, 1 \mathrm{H}, \mathrm{J}=12.0,5.7 \mathrm{MHz}, 5-\mathrm{H}), 6.820-7.015(\mathrm{~m}, 4 \mathrm{H}$, Ar-H), 7.432-7.658 (m, 4H, Ar-H), $8.323\left(\mathrm{~s}, 2 \mathrm{H}, \mathrm{NH}_{2}\right) ;{ }^{13} \mathrm{C} \mathrm{NMR}\left(\mathrm{CDCl}_{3}, \delta \mathrm{ppm}\right): 40.1(2 \mathrm{C}$, 
$\left.\mathrm{N}\left(\mathrm{CH}_{3}\right)_{2}\right), 41.2$ (1C, C-4), 69.2 (1C, C-5), 112.6 (2C), 114.5 (2C), 129.2 (2C), 129.5 (2C), 131.7 (1C), 132.6 (1C), 148.1 (1C), 151.3 (1C, C-3), 160.1 (1C), 174.4 (1C, C=S); MS (m/z): $342.1(\mathrm{M}+, 100)$; Anal. Calcd. (found) for $\mathrm{C}_{18} \mathrm{H}_{19} \mathrm{FN}_{4} \mathrm{~S}$ (\%): C, 63.14 (63.01); H, 5.59 (5.56); $\mathrm{N}, 16.36(16.31)$.

3.1.10. 3-(4-Chlorophenyl)-5-(4-(dimethylamino)phenyl)-4,5-dihydro-1H-pyrazole-1carbothioamide, $5 \mathrm{c}$.

Obtained from 1-(4-chlorophenyl)-3-(4-(dimethylamino)phenyl)prop-2-en-1-one, 3c $(1.43 \mathrm{~g}, 5 \mathrm{mmol})$ and hydrazinecarbothioamide hydrochloride, $4(0.64 \mathrm{~g}, 5 \mathrm{mmol}) ;{ }^{1} \mathrm{H} \mathrm{NMR}$ $\left(\mathrm{CDCl}_{3}, \delta \mathrm{ppm}\right): 2.988\left(\mathrm{~s}, 6 \mathrm{H}, \mathrm{CH}_{3}\right), 3.642\left(\mathrm{dd}, 1 \mathrm{H}, \mathrm{J}=6.1,16.3 \mathrm{MHz}, 4-\mathrm{H}_{\mathrm{a}}\right), 3.948(\mathrm{dd}, 1 \mathrm{H}, \mathrm{J}=$ $\left.18.0,6.0 \mathrm{MHz}, 4-\mathrm{H}_{\mathrm{b}}\right), 4.246\left(\mathrm{dd}, 1 \mathrm{H}, \mathrm{J}=13.6,5.6 \mathrm{MHz}, \mathrm{C}_{5}-\mathrm{H}\right), 6.888-7.070(\mathrm{~m}, 4 \mathrm{H}, \quad$ Ar-H), 7.408-7.655 (m, 4H, Ar-H), $8.290\left(\mathrm{~s}, 2 \mathrm{H}, \mathrm{NH}_{2}\right) ;{ }^{13} \mathrm{C} \mathrm{NMR}\left(\mathrm{CDCl}_{3}, \delta \mathrm{ppm}\right): 39.8(2 \mathrm{C}$, $\left.\mathrm{N}\left(\mathrm{CH}_{3}\right)_{2}\right), 42.6$ (1C, C-4), 68.4 (1C, C-5), 110.7 (2C), 128.7 (2C), 129.8 (2C), 130.1 (2C), 131.3 (1C), 133.8 (1C), 135.4 (1C), 149.3 (1C), 153.7 (1C, C-3), 176.1 (1C, C=S); MS (m/z): $360.0(\mathrm{M}+2,32)$; $358.1(\mathrm{M}+, 100)$; Anal. Calcd. (found) for $\mathrm{C}_{18} \mathrm{H}_{19} \mathrm{ClN}_{4} \mathrm{~S}(\%)$ :, 60.24 (60.13); H, 5.34 (5.30); N, 15.61 (15.56).

3.1.11. 5-(4-(Dimethylamino)phenyl)-3-(p-tolyl)-4,5-dihydro-1H-pyrazole-1-carbothioamide, $5 \mathrm{~d}$.

Obtained from 3-(4-(dimethylamino)phenyl)-1-(p-tolyl)prop-2-en-1-one, 3d (1.33g, 5 $\mathrm{mmol})$ and hydrazinecarbothioamide hydrochloride, 4 (0.64g, $5 \mathrm{mmol}) ;{ }^{1} \mathrm{H} \mathrm{NMR}\left(\mathrm{CDCl}_{3}, \delta\right.$ ppm): 2.382 (s, 3H, $\left.\mathrm{CH}_{3}\right), 2.995\left(\mathrm{~s}, 6 \mathrm{H}, \mathrm{CH}_{3}\right), 3.685$ (dd, $\left.1 \mathrm{H}, \mathrm{J}=7.8,18.5 \mathrm{MHz}, 4-\mathrm{H}_{\mathrm{a}}\right), 3.980$ $\left(\mathrm{dd}, 1 \mathrm{H}, \mathrm{J}=17.7,5.5 \mathrm{MHz}, 4-\mathrm{H}_{\mathrm{b}}\right), 4.270$ (dd, 1H, J= 11.3, 6.6MHz, $\left.\mathrm{C}_{5}-\mathrm{H}\right), 6.902-7.060$ (m, 4H, $\mathrm{Ar}-\mathrm{H}), 7.322-7.515(\mathrm{~m}, 4 \mathrm{H}, \mathrm{Ar}-\mathrm{H}), 8.182\left(\mathrm{~s}, 2 \mathrm{H}, \mathrm{NH}_{2}\right) ;{ }^{13} \mathrm{C} \mathrm{NMR}\left(\mathrm{CDCl}_{3}, \delta \mathrm{ppm}\right): 20.9(1 \mathrm{C}$, $\left.\mathrm{CH}_{3}\right), 40.4$ (2C, $\left.\mathrm{N}\left(\mathrm{CH}_{3}\right)_{2}\right), 42.6$ (1C, C-4), 69.4 (1C, C-5), $110.8(2 \mathrm{C}), 127.6(2 \mathrm{C}), 128.5(2 \mathrm{C})$, 129.1 (2C), 131.4 (1C), 134.1 (1C), 139.9 (1C), 147.7 (1C), 152.0 (1C, C-3), 177.5 (1C, C=S); MS (m/z): $338.2(\mathrm{M}+, 100)$; Anal. Calcd. (found) for $\mathrm{C}_{19} \mathrm{H}_{22} \mathrm{~N}_{4} \mathrm{~S}$ (\%): C, 67.42 (67.35); H, 6.55 (6.51); N, 16.55 (16.50).

3.1.12. 5-(4-(Dimethylamino)phenyl)-3-(3-methoxyphenyl)-4,5-dihydro-1H-pyrazole-1carbothioamide, $5 \mathrm{e}$.

Obtained from 3-(4-(dimethylamino)phenyl)-1-(3-methoxyphenyl)prop-2-en-1-one, 3e $(1.40 \mathrm{~g}, 5 \mathrm{mmol})$ and hydrazinecarbothioamide hydrochloride, $4(0.64 \mathrm{~g}, 5 \mathrm{mmol}) ;{ }^{1} \mathrm{H} \mathrm{NMR}$ $\left(\mathrm{CDCl}_{3}, \delta \mathrm{ppm}\right): 3.012\left(\mathrm{~s}, 6 \mathrm{H}, \mathrm{CH}_{3}\right), 3.655\left(\mathrm{dd}, 1 \mathrm{H}, \mathrm{J}=10.6,17.5 \mathrm{MHz}, 4-\mathrm{H}_{\mathrm{a}}\right), 3.835(\mathrm{~s}, 3 \mathrm{H}$, $\left.\mathrm{OCH}_{3}\right), 3.960$ (dd, $\left.1 \mathrm{H}, \mathrm{J}=18.2,7.6 \mathrm{MHz}, 4-\mathrm{H}_{\mathrm{b}}\right), 4.330(\mathrm{dd}, 1 \mathrm{H}, \mathrm{J}=11.9,6.9 \mathrm{MHz}, 5-\mathrm{H}), 6.978-$ $7.109(\mathrm{~m}, 4 \mathrm{H}, \mathrm{Ar}-\mathrm{H}), 7.538-7.812(\mathrm{~m}, 4 \mathrm{H}, \mathrm{Ar}-\mathrm{H}), 8.216\left(\mathrm{~s}, 2 \mathrm{H}, \mathrm{NH}_{2}\right) ;{ }^{13} \mathrm{C} \mathrm{NMR}\left(\mathrm{CDCl}_{3}, \delta\right.$ ppm): 39.0 (2C, $\left.\mathrm{N}\left(\mathrm{CH}_{3}\right)_{2}\right), 40.9$ (1C, C-4), $55.3\left(1 \mathrm{C}, \mathrm{OCH}_{3}\right), 67.7$ (1C, C-5), $112.2(2 \mathrm{C}), 113.9$ (1C), 116.3 (1C), 120.1 (1C), 129.5 (1C), 129.5 (2C), 132.3 (1C), 134.7 (1C), 149.0 (1C), 152.7 (1C, C-3), 159.5 (1C), 175.1 (1C, C=S); MS (m/z): 354.2 (M+, 100); Anal. Calcd. (found) for $\mathrm{C}_{19} \mathrm{H}_{22} \mathrm{~N}_{4} \mathrm{OS}$ (\%): C, 64.38 (64.30); H, 6.26 (6.22); N, 15.81 (15.76).

3.1.13. 5-(4-(Dimethylamino)phenyl)-3-(4-methoxyphenyl)-4,5-dihydro-1H-pyrazole-1carbothioamide, $5 f$.

Obtained from 3-(4-(dimethylamino)phenyl)-1-(4-methoxyphenyl)prop-2-en-1-one, 3f $(1.41 \mathrm{~g}, 5 \mathrm{mmol})$ and hydrazinecarbothioamide hydrochloride, 4 (0.64g, $5 \mathrm{mmol}) ;{ }^{1} \mathrm{H}$ NMR 
$\left(\mathrm{CDCl}_{3}, \delta \mathrm{ppm}\right): 3.006\left(\mathrm{~s}, 6 \mathrm{H}, \mathrm{CH}_{3}\right), 3.661\left(\mathrm{dd}, 1 \mathrm{H}, \mathrm{J}=10.2,17.4 \mathrm{MHz}, 4-\mathrm{H}_{\mathrm{a}}\right), 3.826(\mathrm{~s}, 3 \mathrm{H}$, $\left.\mathrm{OCH}_{3}\right), 3.955\left(\mathrm{dd}, 1 \mathrm{H}, \mathrm{J}=18.0,7.3 \mathrm{MHz}, 4-\mathrm{H}_{\mathrm{b}}\right), 4.322(\mathrm{dd}, 1 \mathrm{H}, \mathrm{J}=11.7,6.8 \mathrm{MHz}, 5-\mathrm{H}), 6.965-$ 7.129 (m, 6H, Ar-H), 7.821-7.904 (m, 2H, Ar-H), 8.209 (s, 2H, $\left.\mathrm{NH}_{2}\right) ;{ }^{13} \mathrm{C} \mathrm{NMR}\left(\mathrm{CDCl}_{3}, \delta\right.$ ppm): 40.9 (2C, $\left.\mathrm{N}\left(\mathrm{CH}_{3}\right)_{2}\right), 42.1$ (1C, C-4), 55.5 (1C, $\left.\mathrm{OCH}_{3}\right), 69.7$ (1C, C-5), $112.8(2 \mathrm{C}), 115.5$ (2C), 127.9 (1C), 128.6 (2C), 129.3 (2C), 130.8 (1C), 147.6 (1C), 149.2 (1C), 151.9 (1C, C-3), 176.7 (1C, C=S); MS (m/z): $354.1(\mathrm{M}+, 100)$; Anal. Calcd. (found) for $\mathrm{C}_{19} \mathrm{H}_{22} \mathrm{~N}_{4} \mathrm{OS}(\%)$ : $\mathrm{C}$, 64.38 (64.30); H, 6.26 (6.21); N, 15.81 (15.76).

3.1.14. 3-(3,4-Dimethoxyphenyl)-5-(4-(dimethylamino)phenyl)-4,5-dihydro-1H-pyrazole-1carbothioamide, $5 \mathrm{~g}$.

Obtained from 1-(3,4-dimethoxyphenyl)-3-(4-(dimethylamino)phenyl)prop-2-en-1one, $3 \mathrm{~g}(1.56 \mathrm{~g}, 5 \mathrm{mmol})$ and hydrazinecarbothioamide hydrochloride, $4(0.64 \mathrm{~g}, 5 \mathrm{mmol}) ;{ }^{1} \mathrm{H}$ NMR $\left(\mathrm{CDCl}_{3}, \delta \mathrm{ppm}\right): 3.101\left(\mathrm{~s}, 6 \mathrm{H}, \mathrm{CH}_{3}\right), 3.671\left(\mathrm{dd}, 1 \mathrm{H}, \mathrm{J}=8.3,16.9 \mathrm{MHz}, 4-\mathrm{H}_{\mathrm{a}}\right), 3.840$ (s, $\left.6 \mathrm{H}, \mathrm{OCH}_{3}\right), 3.928\left(\mathrm{dd}, 1 \mathrm{H}, \mathrm{J}=16.0,6.3 \mathrm{MHz}, 4-\mathrm{H}_{\mathrm{b}}\right), 4.314(\mathrm{dd}, 1 \mathrm{H}, \mathrm{J}=12.2,5.9 \mathrm{MHz}, 5-\mathrm{H})$, 6.870-7.078 (m, 5H, Ar-H), 7.412-7.536 (m, 2H, Ar-H), 8.305 (s, 2H, NH $)$; ${ }^{13} \mathrm{C}$ NMR $\left(\mathrm{CDCl}_{3}, \delta \mathrm{ppm}\right): 39.8\left(2 \mathrm{C}, \mathrm{N}\left(\mathrm{CH}_{3}\right)_{2}\right), 42.0(1 \mathrm{C}, \mathrm{C}-4), 55.4\left(2 \mathrm{C}, \mathrm{OCH}_{3}\right), 68.9(1 \mathrm{C}, \mathrm{C}-5), 109.1$ (1C), 112.8 (2C), 114.4 (1C), 121.9 (1C), 127.0 (1C), 128.8 (2C), 132.6 (1C), 148.3 (1C), 147.7 (1C), 149.4 (1C), 153.0 (1C, C-3), 175.3 (1C, C=S); MS (m/z): 384.0 (M+, 100); Anal. Calcd. (found) for $\mathrm{C}_{20} \mathrm{H}_{24} \mathrm{~N}_{4} \mathrm{O}_{2} \mathrm{~S}(\%)$ : C, $62.48(62.41) ; \mathrm{H}, 6.29(6.25) ; \mathrm{N}, 14.57$ (14.52).

\subsection{Chemistry.}

Initially, the intermediate 1-aryl-3-(4-(dimethylamino)phenyl)prop-2-en-1-ones, 3(ag), were prepared through the base catalyzed Claisen-Schmidt condensation of 4(dimethylamino)benzaldehyde 1, and substituted acetophenones 2(a-g). Then, amberlyst-15 catalyzed $(3+2)$ annulation reaction of $3(\mathrm{a}-\mathrm{g})$ with hydrazinecarbothioamide hydrochloride, 4 in acetonitrile at room temperature produced pyrazole carbothioamide derivatives $5(\mathrm{a}-\mathrm{g})$ in good yields. The reaction was also performed under conventional reflux conditions in acetic acid medium (Fig. 1) (Table 1). The amberlyst-15 catalyst, recovered using the solvent ethyl acetate, was efficient for four consecutive similar experiments [42]. The structures of synthesized new compounds were confirmed by spectroscopic analysis.

The ${ }^{1} \mathrm{H}$ NMR spectra of compounds 3(a-g) recorded on $400 \mathrm{MHz}$ Agilent-NMR spectrometer show a singlet in the range of $\delta 3.005-3.036 \mathrm{ppm}$ for six $\mathrm{N}\left(\mathrm{CH}_{3}\right)_{2}$ protons, two doublets in the range of $\delta 6.721-6.992(\mathrm{~J}=14.6-18.2 \mathrm{MHz}) \mathrm{ppm}$ for $=\mathrm{CH}$, and $\delta 8.205-8.355$ (13.5-17.8MHz) ppm for $\mathrm{CH}=$ protons. The constant coupling values in the range of $\mathrm{J}=14.6$ 18.2 $\mathrm{MHz}$ and $\mathrm{J}=13.5-17.8 \mathrm{MHz}$ of alkenyl protons indicate the trans configuration around the $\mathrm{C}=\mathrm{C}$ bond. The compounds $5(\mathrm{a}-\mathrm{g})$ show three doublet of doublets in the range $\delta 3.614-3.685$ $(\mathrm{J}=6.3-10.5,16.3-18.5 \mathrm{MHz}), 3.910-3.980(\mathrm{~J}=16.0-19.4,5.5-7.6 \mathrm{MHz})$, and 4.215-4.322 $(\mathrm{J}=$ 11.3-13.6, 5.6-7.0MHz) for 4- $\mathrm{H}_{\mathrm{a}}, 4-\mathrm{H}_{\mathrm{b}}$, and 5-H, protons respectively. Singlets in the range of $\delta$ 2.988-3.101 ppm for six $\mathrm{N}\left(\mathrm{CH}_{3}\right)_{2}$, and $\delta$ 8.182-8.345 ppm for two $\mathrm{NH}_{2}$ protons. Furthermore, compounds 3(a-g) and 5(a-g) show the signals due to substituent and aromatic protons in the respective absorption region. The absence of signals due to alkenyl protons of 3(a-g) and ABX type coupling of methylene protons of pyrazole ring confirm $(3+2)$ annulation. 
<smiles>[R]c1ccc(C(=O)/C=C/c2ccc(N(C)C)cc2)cc1[R19]</smiles>

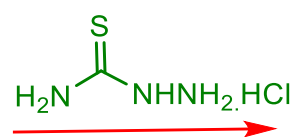

(i) and/or (ii)

Reagents and conditions:<smiles>[R]c1ccc(C2=NN(C(N)=S)C(c3ccc(N(C)C)cc3)C2)cc1[R]</smiles>

4 a) $\mathrm{R}=\mathrm{H}, \quad \mathrm{R}^{1}=\mathrm{H}$;

b) $\mathrm{R}=\mathrm{F}, \quad \mathrm{R}^{1}=\mathrm{H}$;

c) $\mathrm{R}=\mathrm{Cl}, \quad \mathrm{R}^{1}=\mathrm{H}$;

d) $\mathrm{R}=\mathrm{CH}_{3}, \quad \mathrm{R}^{1}=\mathrm{H}$;

e) $\mathrm{R}=\mathrm{H}, \quad \mathrm{R}^{1}=\mathrm{OCH}_{3}$;

f) $\mathrm{R}=\mathrm{OCH}_{3}, \mathrm{R}^{1}=\mathrm{H}$;

g) $\mathrm{R}=\mathrm{OCH}_{3}, \mathrm{R}^{1}=\mathrm{OCH}_{3}$.

(i) Amberlyst-15 (10\%, w/w)/acetonitrile, rt, 1-2 h.; (ii) Acetic acid (30\%), reflux, 2-3 h.

Figure 1. Synthesis of pyrazole carbothioamide derivatives, 4(a-g).

Table 1. Reaction time and yields of amberlyst-15 mediated and conventional synthesis.

\begin{tabular}{l|c|c|c|c|c}
\multirow{2}{*}{ Entry } & Amberlyst-15 mediated method & \multicolumn{2}{c}{ Conventional method } & \multirow{2}{*}{$\begin{array}{c}\text { Melting } \\
\text { Point }\left({ }^{\circ} \mathbf{C}\right)\end{array}$} \\
\cline { 2 - 5 } & Time (Min) & Yield (\%) & Time (Min) & Yield (\%) & $121-123$ \\
\hline $\mathbf{4 a}$ & 95 & 84 & 155 & 82 & $133-135$ \\
\hline $\mathbf{4 b}$ & 90 & 81 & 140 & 76 & $146-147$ \\
\hline $\mathbf{4 c}$ & 60 & 85 & 120 & 61 & $115-117$ \\
\hline $\mathbf{4 d}$ & 115 & 70 & 175 & 60 & $96-98$ \\
\hline $\mathbf{4 e}$ & 120 & 66 & 180 & 71 & $108-110$ \\
\hline $\mathbf{4 f}$ & 75 & 78 & 140 & 70 & $155-157$
\end{tabular}

The ${ }^{13} \mathrm{C}$ NMR spectra of compounds $3(\mathrm{a}-\mathrm{g})$ recorded on $100 \mathrm{MHz}$ Agilent-NMR spectrometer show the carbon resonance signals in the range of $\delta 39.5-41.6 \mathrm{ppm}, 121.2-124.4$ ppm, 143.1-144.8 ppm, and $\delta$ 187.2-190.2 ppm, for two $\mathrm{N}\left(\mathrm{CH}_{3}\right)_{2}$, alkenyl $\alpha$-, alkenyl $\beta$-, and carbonyl carbons, respectively. The compounds $5(\mathrm{a}-\mathrm{g})$ show carbon resonance signals in the range of $\delta$ 40.9-43.8 ppm, 67.7-69.9 ppm, 151.3-153.7 ppm for C-4, C-5, and C-3 carbons of newly formed pyrazole ring. The $\mathrm{C}=\mathrm{S}$ carbons were absorbed in the range of $\delta$ 174.4-177.5 ppm, while the signals that appear in the range of $\delta 39.8-41.8 \mathrm{ppm}$ were unambiguously assigned to $\mathrm{N}\left(\mathrm{CH}_{3}\right)_{2}$ carbons. The compounds 3(a-g) and 5(a-g) show the substituent and aromatic carbon signals in the respective absorption region. The mass spectra of compounds 3(a-g) and 5(a-g) recorded on ESI/APCI-Hybrid Quadrupole, Synapt G2 HDMS ACQUITY UPLC model spectrometer show molecular ion base peaks corresponding to their molecular masses. However, the compounds $3 \mathrm{c}$ and $5 \mathrm{c}$ show an $\mathrm{M}+2$ peak at $\mathrm{m} / \mathrm{z} 360$ with a relative abundance of $32 \%$ due to ${ }^{37} \mathrm{Cl}$ isotopic mass. All the compounds show satisfactory elemental analyses obtained on a Thermo Finnigan Flash EA 1112 CHN analyzer compared with theoretical values.

\subsection{Antimicrobial activity.}

\subsubsection{Antifungal activity.}

The results of antifungal activities of the synthesized compounds 5(a-g) measured as minimal inhibitory concentrations were given in Table 2.

Table 2. Minimum inhibitory concentrations $(\mu \mathrm{g} / \mathrm{mL})$ of pyrazole derivatives $\mathbf{5}(\mathbf{a}-\mathbf{g})$ against fungi species.

\begin{tabular}{c|c|c|c} 
Compound & A. niger & A. flavus & C. albicans \\
\hline 5a & $25.0 \pm 0.75$ & $25.0 \pm 0.65$ & $70.0 \pm 0.50$ \\
\hline $\mathbf{5 b}$ & $20.0 \pm 0.55$ & $15.0 \pm 0.60$ & $60.0 \pm 0.90$
\end{tabular}




\begin{tabular}{c|c|c|c} 
Compound & A. niger & A. flavus & C. albicans \\
\hline $\mathbf{5 c}$ & $15.0 \pm 0.80$ & $10.0 \pm 0.65$ & $65.0 \pm 1.05$ \\
\hline $\mathbf{5 d}$ & $25.0 \pm 1.00$ & $25.0 \pm 0.95$ & $60.0 \pm 1.05$ \\
\hline $\mathbf{5 e}$ & $45.0 \pm 0.75$ & $50.0 \pm 1.20$ & $75.0 \pm 1.25$ \\
\hline $\mathbf{5 f}$ & $30.0 \pm 1.00$ & $30.0 \pm 1.15$ & $80.0 \pm 1.20$ \\
\hline $\mathbf{5 g}$ & $40.0 \pm 0.75$ & $50.0 \pm 0.80$ & $90.0 \pm 1.10$ \\
\hline Nystatin & $25.0 \pm 0.75$ & $25.0 \pm 0.25$ & $20.0 \pm 0.50$ \\
Values are mean \pm SD of three replicates; nystatin - positive control.
\end{tabular}

The synthesized compounds displayed varying effects on the tested fungi species. All the compounds show poorer inhibition against $C$. albicans with MIC values in the range of 60.0-90.00 $\mu \mathrm{g} / \mathrm{mL}$ concentrations comparable with the standard nystatin (MIC-20.0 $\mu \mathrm{g} / \mathrm{mL}$ ), however, they show moderate to good inhibition against $A$. niger, and A. flavus. The compounds 3-(4-chlorophenyl)-5-(4-(dimethylamino)phenyl)-4,5-dihydro-1H-pyrazole-1carbothioamide, 5c, and 5-(4-(dimethylamino)phenyl)-3-(4-fluorophenyl)-4,5-dihydro-1 $\mathrm{H}$ pyrazole-1-carbothioamide, 5b show excellent inhibition against $A$. niger $(15$, and $20 \mu \mathrm{g} / \mathrm{mL})$, and A. flavus (20, and $15 \mu \mathrm{g} / \mathrm{mL}$ ); the compounds 5-(4-(dimethylamino)phenyl)-3-phenyl-4,5dihydro- $1 H$-pyrazole-1-carbothioamide, 5a， 5-(4-(dimethylamino)phenyl)-3-(p-tolyl)-4,5dihydro-1H-pyrazole-1-carbothioamide, $5 \mathrm{~d}$ and 5-(4-(dimethylamino)phenyl)-3-(4methoxyphenyl)-4,5-dihydro-1H-pyrazole-1-carbothioamide, 5f found good against $A$. niger $(25,25$, and $30 \mu \mathrm{g} / \mathrm{mL})$, and A. flavus $(25,25$, and $30 \mu \mathrm{g} / \mathrm{mL})$ concentrations. The compounds 5-(4-(dimethylamino)phenyl)-3-(3-methoxyphenyl)-4,5-dihydro-1 $H$-pyrazole-1-

carbothioamide, 5e and 3-(3,4-dimethoxyphenyl)-5-(4-(dimethylamino)phenyl)-4,5-dihydro$1 H$-pyrazole-1-carbothioamide, $5 \mathrm{~g}$ have moderate activities against $A$. niger (45, and 40 $\mu \mathrm{g} / \mathrm{mL}$ ), and A. flavus (50, and $50 \mu \mathrm{g} / \mathrm{mL}$ ) comparable with the standard nystatin (MIC-25.0 $\mu \mathrm{g} / \mathrm{mL}$ ). The preliminary assay results indicate that the compounds $5 \mathrm{c}$ and $5 \mathrm{~b}$ of the series could be lead molecules against $A$. niger and A. flavus organisms.

\subsubsection{Antibacterial activity.}

The results of the compounds 5(a-g) antibacterial activities measured as minimal inhibitory concentrations were summarized in Table 3.

Table 3. Minimum inhibitory concentrations $(\mu \mathrm{g} / \mathrm{mL})$ of pyrazole derivatives $5(\mathrm{a}-\mathrm{g})$ against bacteria species.

\begin{tabular}{l|l|l|l} 
Compound & S. aureus & $\boldsymbol{E}$ coli & B. subtilis \\
\hline $\mathbf{5 a}$ & $15.0 \pm 0.55$ & $15.0 \pm 0.70$ & $20.0 \pm 0.60$ \\
\hline $\mathbf{5 b}$ & $15.0 \pm 0.80$ & $15.0 \pm 0.55$ & $25.0 \pm 0.75$ \\
\hline $\mathbf{5 c}$ & $10.0 \pm 0.65$ & $10.0 \pm 0.60$ & $15.0 \pm 0.80$ \\
\hline $\mathbf{5 d}$ & $25.0 \pm 0.85$ & $30.0 \pm 1.10$ & $30.0 \pm 1.05$ \\
\hline $\mathbf{5 e}$ & $>100$ & $>100$ & $>100$ \\
\hline $\mathbf{5 f}$ & $30.0 \pm 1.20$ & $25.0 \pm 0.70$ & $35.0 \pm 1.10$ \\
\hline $\mathbf{5 g}$ & $>100$ & $>100$ & $>100$ \\
\hline $\begin{array}{l}\text { Ciprofloxacin } \\
\text { Values are mean } \pm \text { SD of three replicates; ciprofloxacin - positive control }\end{array}$
\end{tabular}

The preliminary assessment results show that $5(\mathrm{a}-\mathrm{g})$ compounds exert varying effects on the tested bacteria species. Amongst the series, the compounds 5a, 5b, and 5c displayed excellent inhibition with lesser MIC values against $S$. aureus $(15,15$, and $10 \mu \mathrm{g} / \mathrm{mL})$, E. coli $(15,15$, and $10 \mu \mathrm{g} / \mathrm{mL})$, and B. subtilis $(20,25$, and $15 \mu \mathrm{g} / \mathrm{mL})$ comparable with the standard ciprofloxacin $(20,20$, and $30 \mu \mathrm{g} / \mathrm{mL})$. The compounds $5 \mathrm{~d}$ and $5 \mathrm{e}$ show good activities by inhibiting spore germination of $S$. aureus $(25$, and $30 \mu \mathrm{g} / \mathrm{mL})$, E. coli (30 and $25 \mu \mathrm{g} / \mathrm{mL})$, and B. subtilis (30, and $35 \mu \mathrm{g} / \mathrm{mL}$ ), respectively. However, it was observed that the compounds $5 \mathrm{e}$ and $5 \mathrm{~g}$ having a methoxy substitution in the phenyl ring were found inactive even at $100 \mu \mathrm{g} / \mathrm{mL}$ 
concentrations. From the results, it was found that the compounds $5 \mathrm{a}, 5 \mathrm{~b}$, and $5 \mathrm{c}$ of the series might be future lead bacterial agents.

\section{Conclusions}

To sum up, the current work demonstrates the synthesis of series of pyrazole carbothioamide derivatives through $(3+2)$ annulation reaction of chalcones with hydrazinecarbothioamide hydrochloride catalyzed by an amberlyst-15; the method is reliable and efficient for the synthesis of pyrazoles. The in vitro antifungal and antibacterial activity assay results indicate the noticeable activity of the synthesized new compounds. The compounds $5 \mathrm{a}, 5 \mathrm{~b}$, and $5 \mathrm{c}$ displayed excellent inhibition with least MIC values against the tested $S$. aureus $(15,15$, and $10 \mu \mathrm{g} / \mathrm{mL})$, E. coli $(15,15$, and $10 \mu \mathrm{g} / \mathrm{mL}), B$. subtilis $(20,25$, and $15 \mu \mathrm{g} / \mathrm{mL}$ ); 5b, and 5c against $A$. niger (15, and $20 \mu \mathrm{g} / \mathrm{mL}$ ), and A. flavus (20, and $15 \mu \mathrm{g} / \mathrm{mL}$ ), the results of which were comparable with the standards employed. Therefore, these pyrazole carbothioamides are posited to be the lead candidates for developing new antimicrobial drugs.

\section{Funding}

This research received no external funding.

\section{Acknowledgments}

The authors are grateful to the IOE Instrumentation Facility, Vijnana Bhavana, University of Mysore, for spectral analysis.

\section{Conflicts of Interest}

The authors declare no conflict of interest.

\section{References}

1. Alekshun, M.N.; Levy, S.B. Molecular mechanisms of antibacterial multidrug resistance. Cell 2007, 128, 1037-1050, https://doi.org/10.1016/j.cell.2007.03.004.

2. Vagish, C.B.; Kumar, A.D.; Kumara, K.; Vivek, H.K.; Renuka, N.; Lokanath, N.K.; Kumar, K.A. Environmentally benign synthesis of substituted pyrazoles as potent antioxidant agents, characterization and docking studies. Journal of Iranian Chemical Society 2021, 18, 479-493, https://doi.org/10.1007/s13738-02002042-6.

3. Yi, F.; Zhao, W.; Wang, Z.; Bi, X. Silver-mediated $[3+2]$ cycloaddition of alkynes and $N$ isocyanoiminotriphenylphosphorane: Access to monosubstituted pyrazoles. Organic Letters 2019, 21 , 3158 3161, https://doi.org/10.1021/acs.orglett.9b00860.

4. Lellek, V.; Chen, C.-Y.; Yang, W.; Liu, J.; Ji, X.; Faessler, R. An efficient synthesis of substituted pyrazoles from one-pot reaction of ketones, aldehydes, and hydrazine monohydrochloride. Synlett 2018, 29, 1071-1075, https://doi.org/10.1055/s-0036-1591941.

5. Prabhashankar, J.; Govindappa, V.K.; Kariyappa, A.K. Synthesis of 3,4-diaryl-1-phenyl-4,5-dihydro-1Hpyrazole-5-carbonitriles via 1,3-dipolar cycloaddition reactions. Turkish Journal of Chemistry 2013, 37, 853857, https://doi.org/10.3906/kim-1209-52.

6. Govindaraju, M.; Vasanth Kumar, G.; Mylarappa, B.N.; Ajay Kumar, K. Synthesis of 8-(5-aryl-4-octyl-2phenyl-3,4-dihydro-2H-pyrazol-3-yl)-octanoic acid ethyl esters via 1, 3-dipolar cycloaddition reaction. IOSR Journal of Applied Chemistry 2012, 2, 1-4, https://doi.org/10.9790/5736-0210104.

7. Govindaraju, M.; Vasanth Kumar, G.; Pavithra, G.; Harish Nayaka, M.A.; Mylarappa, B.N.; Ajay Kumar, K. Evaluation of new tetra substituted pyrazolines for their antimicrobial and antioxidant activity; Structureactivity relationship. IOSR Journal of Pharmacy and Biological Sciences 2012, 2, 30-34, https://doi.org/10.9790/3008-0263032.

8. Zhang, Y.; Liu, J.; Jia, X. Phosphine-free [3+2] cycloaddition of propargylamines with dialkyl azodicarboxylates: an efficient access to pyrazole backbone. Synthesis 2018, 50, 3499-3505, https://doi.org/10.1055/s-0037-1610168. 
9. Schmitt, D.C.; Taylor, A.P.; Flick, A.C.; Kyne, Jr.R.E. Synthesis of pyrazoles from 1,3-diols via hydrogen transfer catalysis. Organic Letters 2015, 17, 1405-1408, https://doi.org/10.1021/acs.orglett.5b00266.

10. Achutha, D.K.; Kumara, K.; Shivalingegowda, N.; Krishnappagowda, L.N.; Kariyappa, A.K.; Synthesis of ethyl 5-(4-chlorophenyl)-3-methyl-1-phenyl-1H-pyrazole-4-carboxylate by an unusual protocol: Crystal and molecular structure, Hirshfeld surface analysis. Chemical Data Collections 2017, 9, 89-97, https://doi.org/10.1016/j.cdc.2017.04.002.

11. Prabhudeva, M.G.; Prabhuswamy, M.; Kumar, A.D.; Lokanath, N.K.; Naveen, S.; Kumar, K.A. Synthesis, crystal and molecular structure and antimicrobial studies of 1-(3-chlorophenyl)-3-(5-chlorothiophen-2-yl)-5phenyl-4,5-dihydro-1H-pyrazole. Chemical Data Collections 2017, 9, 80-88, https://doi.org/10.1016/j.cdc.2017.03.005.

12. Prabhudeva, M.G.; Naveen, S.; Raghavendra, K.R.; Kumar, A.D.; Kumara, K.; Lokanath, N.K.; Kumar, K.A. 1-(3-Chlorophenyl)-5-(4-chlorophenyl)-3-(5-chlorothiophen-2-yl)-4,5-dihydro-1H-pyrazole. IUCrData 2017, 2, https://doi.org/10.1107/S2414314616020484.

13. Naveen, S.; Kumara, K.; Kumar, A.D.; Kumar, K.A.; Lokanath, N.K. Structural elucidation and Hirshfeld Surface Analysis of a novel pyrazole derivative: 3-(Benzo[d][1,3]dioxol-5-yl)-1-(3-chlorophenyl)-5-(2,4dichlorophenyl)-4,5-dihydro-1H-pyrazole. Chemical Data Collections 2018, 15, 89-96, https://doi.org/10.1016/j.cdc.2018.04.006.

14. Prabhuswamy, M.; Kumara, K.; Pavithra, G.; Ajay Kumar, K.; Lokanath, N.K. Synthesis, crystal structure and Hirshfeld surface analysis of 5-(3,4-Dimethoxyphenyl)-3-(thiophen-2-yl)-4,5-dihydro-1H-pyrazole-1carboxamide. Chemical Data Collections 2016, 3, 26-35, https://doi.org/10.1016/j.cdc.2016.06.004.

15. Kumara, K.; Kumar, A.D.; Naveen, S.; Kumar, K.A.; Lokanath, N.K. Synthesis, spectral characterization and X-ray crystal structure studies of 3-(benzo[d][1,3]dioxol-5-yl)-5-(3-methylthiophen-2-yl)-4,5-dihydro-1Hpyrazole-1-carboxamide: Hirshfeld surface, DFT and thermal analysis. Journal of Molecular Structure 2018, 1161, 285-298, https://doi.org/10.1016/j.molstruc.2018.02.068.

16. Kumara, K.; Kumar, A.D.; Kumar, K.A.; Lokanath, N.K. Synthesis, spectral and X-ray crystal structure of 3-(3-methoxyphenyl)-5-(3-methylthiophen-2-yl)-4,5-dihydro-1H-pyrazole-1-carboxamide: Hirshfeld surface, DFT calculations and thermo-optical studies. Chemical Data Collections 2018, 13, 40-59, https://doi.org/10.1016/j.cdc.2018.01.001.

17. Naveen, S.; Pavithra, G.; Abdoh, M.; Ajay Kumar, K.; Warad, I.; Lokanath, N.K. Crystal structure of 3(thiophene-2-yl)-5-p-tolyl-4,5-dihydro-1H-pyrazole-1-carbothioamide. Acta Crystallographica 2015, E71, 763-765, https://doi.org/10.1107/S2056989015010774.

18. Achutha, D.K.; Vagish, C.B.; Renuka, N.; Lokeshwari, D.M.; Kariyappa, A.K. Green synthesis of novel pyrazoline carbothioamides: A potent antimicrobial and antioxidant agents. Chemical Data Collections 2020, 28, https://doi.org/10.1016/j.cdc.2020.100445.

19. Dileep Kumar, A.; Vagish, C.B.; Lokeshwari, D.M.; Sowmya, R.; Ajay Kumar, K. Design, synthesis, characterization, evaluation for anticancer and citotoxic properties of new pyrazole carbothioamides. Asian Journal of Organic and Medicinal Chemistry 2021, 6, 53-58.

20. Kumara, K.; Shivalingegowda, N.; Mahadevaswamy, L.D.; Kariyappa, A.K.; Lokanath, N.K. Crystal structure studies and Hirshfeld surface analysis of 5-(4-methoxyphenyl)-3-(thiophen-2-yl)-4,5-dihydro-1Hpyrazole-1-carbothioamide. Chemical Data Collections 2017, 9-10, 251-262, https://doi.org/10.1016/j.cdc.2016.11.006.

21. Bukhari, S.N.A.; Lauro, G.; Jantan, I.; Bifulco, G.; Amjad, M.W. Pharmacological evaluation and docking studies of $\alpha, \beta$-unsaturated carbonyl based synthetic compounds as inhibitors of secretory phospholipase $\mathrm{A}_{2}$, cyclooxygenases, lipoxygenase and proinflammatory cytokines, Bioorganic \& Medicinal Chemistry 2014, 22, 4151-4161, https://doi.org/10.1016/j.bmc.2014.05.052.

22. Vagish, C.B.; Sowmya, R.; Sudeep, P.; Ajay Kumar, K. Recent developments on the synthetic and biological applications of chalcones-A Review. Biointerface Research in Applied Chemistry 2022, 12, 180-195, https://doi.org/10.33263/BRIAC121.180195.

23. Ajay Kumar, K.; Renuka, N. Thienyl chalcones: Small molecules that play pivotal roles. International Journal of Current Research 2021, 13, 16067-16074, https://doi.org/10.24941/ijcr.40692.02.2021.

24. Vishnu, W.K.; Abheesh, P.; Guruvayoorappan, C. Pyrazole (1, 2-diazole) induce apoptosis in lymphoma cells by targeting BCL-2 and BCL-XL genes and mitigate murine solid tumour development by regulating cyclinD1 and Ki-67 expression. Toxicol Applied Pharmacology 2021, 418, https://doi.org/10.1016/j.taap.2021.115491.

25. Chaudhry, F.; Shahid, W.; Rashida, M.; Ashraf, M.; Munawar, M.A.; Khan, M.A. Synthesis of imidazolepyrazole conjugates bearing aryl spacer and exploring their enzyme inhibition potentials. Bioorganic Chemistry 2021, 108, https://doi.org/10.1016/j.bioorg.2021.104686.

26. Afifi, O.S.; Shaaban, O.G.; Abd El Razik, H.A.; Shams El-Dine, S.El-D.A.; Ashour, F.A.; El-Tombary, A.A.; Abu-Serie, M.M. Synthesis and biological evaluation of purine-pyrazole hybrids incorporating thiazole, thiazolidinone or rhodanine moiety as 15-LOX inhibitors endowed with anticancer and antioxidant potential, Bioorganic Chemistry 2019, 87, 821-837, https://doi.org/10.1016/j.bioorg.2019.03.076. 
27. Zhang, Z.; Min, J.; Chen, M.; Jiang, X.; Xu, Y.; Qin, H.; Tang, W. The structure-based optimization of $\delta$ sultone-fused pyrazoles as selective BuChE inhibitors, European Journal of Medicinal Chemistry 2020, 201, https://doi.org/10.1016/j.ejmech.2020.112273.

28. Shaaban, M.M.; Ragab, H.M.; Akaji, K.; McGeary, R.P.; Bekhit, A.-A.A.; Hussein, W.M.; Kurz, J.L.; Elwakil, B.H.; Bekhit, S.A.; Ibrahim, T.M.; Mahran, M.A.; Bekhit, A.A. Design, synthesis, biological evaluation and in silico studies of certain aryl sulfonyl hydrazones conjugated with 1,3-diaryl pyrazoles as potent metallo- $\beta$-lactamase inhibitors. Bioorganic Chemistry 2020, 105, https://doi.org/10.1016/j.bioorg.2020.104386.

29. Kumar,A.D.; Prabhudeva, M.G.; Bharath, S.; Kumara, K.; Lokanath, N.K.; Kumar, K.A. Design and Amberlyst-15 mediated synthesis of novel thienyl-pyrazole carboxamides that potently inhibit Phospholipase A2 by binding to an allosteric site on the enzyme. Bioorganic Chemistry 2018, 80, 444-452, https://doi.org/10.1016/j.bioorg.2018.06.023.

30. Lokeshwari, D.M.; Achutha, D.K.; Srinivasan, B.; Shivalingegowda, N.; Krishnappagowda, L.N.; Kariyappa, A.K. Synthesis of novel pyrazole analogues with potent affinity anti-inflammatory effect mediated by inhibition of phospholipase A2: Crystallographic, in silico docking and QSAR analysis. Bioorganic Medicinal Chemistry Letters 2017, 27, 3806-3811, https://doi.org/10.1016/j.bmcl.2017.06.063.

31. Hassan, G.S.; Georgey, H.H.; Mohammed, E.Z.; George, R.F.; Mahmoud, W.R.; Omar, F.A. Mechanistic selectivity investigation and 2D-QSAR study of some new antiproliferative pyrazoles and pyrazolopyridines as potential CDK2 inhibitors. European Journal of Medicinal Chemistry 2021, 218, https://doi.org/10.1016/j.ejmech.2021.113389.

32. Kumar, A.D.; Bharath, S.; Dharmappa, R.N.; Naveen, S.; Lokanath, N.K.; Ajay Kumar, K. Design, synthesis and spectroscopic and crystallographic characterisation of novel functionalized pyrazole derivatives: biological evaluation for their cytotoxic, angiogenic and antioxidant activities. Research on Chemical Intermediates 2018, 44, 5635-5652, https://doi.org/10.1007/s11164-018-3445-6.

33. Bennani, F.E.; Doudach, L.; Cherrah, Y.; Ramli, Y.; Karrouchi, K.; Ansar, M.; Faouzi, M.El. A. Overview of recent developments of pyrazole derivatives as an anticancer agent in different cell line. Bioorganic Chemistry 2020, 97, https://doi.org/10.1016/j.bioorg.2019.103470.

34. Kumar, A.D.; Vivek, H.K.; Srinivasan, B.; Naveen, S.; Kumara, K.; Lokanath, N.K.; Byrappa, K.; Kumar, K.A. Design, synthesis, characterization, crystal structure, Hirshfeld surface analysis, DFT calculations, anticancer, angiogenic properties of new pyrazole carboxamide derivatives. Journal of Molecular Structure 2021, 1235, https://doi.org/10.1016/j.molstruc.2021.130271.

35. Gurunanjappa, P.; Kameshwar, V.H.; Kariyappa, A.K. Bioactive formylpyrazole analogues: Synthesis, antimicrobial, antioxidant and molecular docking studies. Asian Journal of Chemistry 2017, 29, 1549-1554, https://doi.org/10.14233/ajchem.2017.20562.

36. Gurunanjappa, P.; Ningappa, M.B.; Kariyappa, A.K. Synthesis of pyrazole fused pyran analogues: Antimicrobial, antioxidant and molecular docking studies. Chemical Data Collections 2016, 5, 1-11, https://doi.org/10.1016/j.cdc.2016.09.002.

37. Patel, B.; Zunk, M.; Grant, G.; Rudrawar, S. Design, synthesis and bioactivity evaluation of novel pyrazole linked phenylthiazole derivatives in context of antibacterial activity. Bioorganic \& Medicinal Chemistry Letters 2021, 39, https://doi.org/10.1016/j.bmcl.2021.127853.

38. Ramyashree, D.; Raghavendra, K.R.; Dileep Kumar, A.; Vagish, C.B.; Ajay Kumar, K. Synthesis, characterization and antimicrobial activities of chalcones and their post transformation to pyrazole derivatives. Asian Journal of Chemistry 2017, 29, 1538-1542, https://doi.org/10.14233/ajchem.2017.20561.

39. Wang, L.; Ma, L.-Y.; Huang, Y.-L.; Zheng, B.-Y. (E)-3-[4-(Dimethylamino)phenyl]-1-(4methylphenyl)prop-2-en-1-one. Acta Crystallographica https://doi.org/10.1107/S1600536809052398.

40. Ajay Kumar, K.; Lokanatha Rai, K.M. U853mesha, K.B. Synthesis and evaluation of antifungal and antibacterial activity of ethyl 3,5-diarylisoxazole-4-carboxylates. Journal of Chemical Research (S) 2001, 436-438, https://doi.org/10.3184/030823401103168389.

41. Mahadevaswamy, L.D.; Kariyappa, A.K. An environmentally benign lemon juice mediated synthesis of novel furan conjugated pyrazole derivatives and their biological evaluation. Pharmaceutical Chemistry Journal 2017, 51, 670-677, https://doi.org/10.1007/s11094-017-1672-6.

42. Prabhudeva, M.G.; Kumara, K.; Kumar, A.D.; Ningappa, M.B.; Lokanath, N.K.; Kumar, K.A. Amberlyst-15 catalyzed synthesis of novel thiophene-pyrazoline derivatives: spectral and crystallographic characterization and anti-inflammatory and antimicrobial evaluation. Research on Chemical Intermediates 2018, 44, 64536468, https://doi.org/10.1007/s11164-018-3501-2. 